\title{
Modeling of Top Scroll Profile Using Equidistant-Curve Approach for a Scroll Compressor
}

\author{
Tao Liu and Zaixin Wu \\ School of Mechanical and Electronical Engineering, Lanzhou University of Technology, 287 Langongping Road, Lanzhou 730050, China \\ Correspondence should be addressed to Tao Liu; cathyliu1999@126.com
}

Received 6 February 2015; Revised 22 May 2015; Accepted 31 May 2015

Academic Editor: Filippo Ubertini

Copyright (C) 2015 T. Liu and Z. Wu. This is an open access article distributed under the Creative Commons Attribution License, which permits unrestricted use, distribution, and reproduction in any medium, provided the original work is properly cited.

\begin{abstract}
Scroll profile plays a key role in determining the performance of a scroll compressor. In this study geometric and dynamic characteristics of the scroll profile are analyzed to investigate the influence of its geometric continuity on property of a scroll compressor. Firstly, scroll profiles are created to redesign the geometry of scroll wrap by using the equidistant-curve approach on the basis of a generation line consisting of involute of circle and circular arc. Subsequently, the geometric and dynamic models of the scroll compressor are established. These models are related to parameters of the generation line of scroll profile and rotation angle of a moving scroll. Lastly, some simulation examples of second-order continuity (SOC) scroll profile are compared with firstorder continuity (FOC) scroll profiles and some important conclusions are obtained. Results show that SOC scroll profile is superior to FOC profile in terms of volume ratio, stability of gas force, and possible leakage loss in a scroll compressor.
\end{abstract}

\section{Introduction}

Scroll compressors are widely used for compressing air or refrigerants in refrigerators and air conditioners. Scroll compressors have the advantage of higher efficiency and lower noise and vibration levels, and they are more reliable because of the smaller number of moving parts required for their operation compared with other types of compressors [1].

One problem when developing a scroll compressor is the design of scroll profile, which is an important factor affecting the performance of the compressor. Many researchers have focused on this subject and done a lot of work [2-7] based on either dynamic analysis or optimum design considerations. Liu et al. [8] presented a graphic and mathematical method for the modification of the start part of scroll profile. Afterwards, Lee and $\mathrm{Wu}$ [9] gave an analytical method to design scroll profile with first-order continuity (FOC). These foregoing researches had focused on interpolating scroll profile by using circular arcs with geometry condition to zero order or FOC for a scroll compressor. Notably, Bush and Beagle [10] put forward a general relationship which governs conjugacy of scroll profiles and proved that the general conditions for conjugacy could assure first- and second-order continuity (SOC). More recently, Gravesen and Henriksen [11] redefined the scroll geometry by radius of curvature as a function of tangent direction. In line with the previous work, Blunier et al. [12] proposed a new way of describing the geometry of the scroll wrap by using an orthogonal reference frame. However, there is little information available in literature about SOC scroll profiles for scroll fluid machines. Furthermore, the approach to generate two meshed profiles with inherent conjugate relationship has not been given publicly. Nevertheless, further research may still be needed.

This paper presents a new design of SOC top scroll profile with circular arcs curves based on the curvature radius function proposed by Gravesen and Henriksen [11]. The generation of scroll wrap is illustrated in detail. The estimations of geometric and dynamic properties are analytically described and implemented by computer simulation. For comparison purposes some simulation examples for SOC and FOC profiles are presented. The results suggest many advantages of SOC scroll profiles over FOC ones.

\section{Generation Line of Scroll Profiles}

The typical profile of scroll compressors is formed by an involute of circle. Involute designs have several advantages: simple formulation, uniform wall thickness, and small force 
vibration. However, there are some limitations. Only profile outside of base circle of the involute can be generated while using this kind of curve. The top profile within base circle is usually formed by interference between a cutter and the scroll component when it is machined. However, the thus formed top profile of two scrolls cannot engage each other, which results in a smaller volume ratio and weaker top scroll wrap of a scroll compressor. In order to overcome these defects, we reconstruct the starting part of the involute with a circular arc going through ordinate origin to form an engaged top profile. As a result, a complete scroll profile including the portion within base circle is obtained and defined as the generation line of scroll profiles, as shown in Figure 1. In the generation line, the circular arc and the involute of circle are jointed at point $P$. The starting tangential angle of circular arc is defined as $\varphi_{1}$ and the ending tangential angle of circular arc is defined as $\varphi_{2}$, and the radius of circular arc is represented as $R_{s}$. In order to assure SOC geometric boundary condition of scroll profiles, the following requirements must be met:

(1) Tangential angle $\varphi_{2}$ should be equal to the tangential angle of involute of circle at point $P$.

(2) The radius of curvature of point $P$ on circular curve should be the same as that on involute of circle.

If the above requirement (2) cannot be satisfied, then the geometric boundary condition for scroll profile can only maintain FOC.

In a scroll compressor the two identical principal components, that is, the fixed and moving scrolls, are positioned so that they share a common center; any arbitrary pair of conjugation points is separated by a distance $R_{\text {or }}$, the rotation radius, directed normally to the respective surfaces. Thus, the generation line and its normal equidistant lines can be applied as scroll profiles that have conjugate relation, as illustrated in Figure 2.

The mathematical model for the generation line of scroll profile is given as

$$
\begin{array}{r}
x=\int_{\varphi_{1}}^{\varphi} \rho(\varphi) \cos \varphi \mathrm{d} \varphi, \\
y=\int_{\varphi_{1}}^{\varphi} \rho(\varphi) \sin \varphi \mathrm{d} \varphi, \\
\left(\varphi \in\left[\varphi_{1}, \varphi_{\max }\right]\right),
\end{array}
$$

where radius of curvature of involute

$$
\rho(\varphi)= \begin{cases}R_{s} & \left(\varphi \in\left[\varphi_{1}, \varphi_{2}\right]\right) \\ a \varphi & \left(\varphi \in\left[\varphi_{2}, \varphi_{\max }\right]\right) .\end{cases}
$$

The equation for outer profile of moving scroll can be represented as follows:

$$
\begin{array}{r}
x_{\mathrm{mo}}=\int_{\varphi_{1}}^{\varphi}\left[\rho(\varphi)-\frac{R_{\mathrm{or}}}{2}\right] \cos \varphi \mathrm{d} \varphi \\
y_{\mathrm{mo}}=\int_{\varphi_{1}}^{\varphi}\left[\rho(\varphi)-\frac{R_{\mathrm{or}}}{2}\right] \sin \varphi \mathrm{d} \varphi \\
\left(\varphi \in\left[\varphi_{1}, \varphi_{\max }\right]\right) .
\end{array}
$$

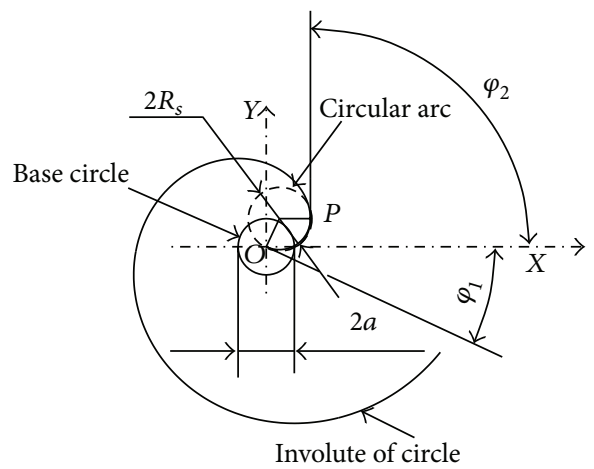

FIgURE 1: Generation line of scroll profile.

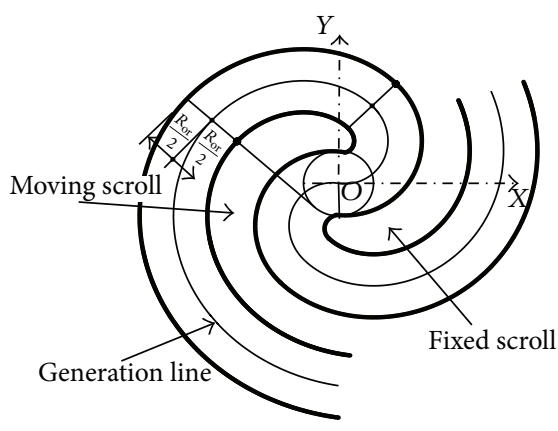

FIGURE 2: Schematic of scroll profile.

The equation for inner profile of fixed scroll can be written as

$$
\begin{array}{r}
x_{\mathrm{fi}}=\int_{\varphi_{1}}^{\varphi}\left[\rho(\varphi)+\frac{R_{\text {or }}}{2}\right] \cos \varphi \mathrm{d} \varphi \\
y_{\mathrm{fi}}=\int_{\varphi_{1}}^{\varphi}\left[\rho(\varphi)+\frac{R_{\mathrm{or}}}{2}\right] \sin \varphi \mathrm{d} \varphi \\
\left(\varphi \in\left[\varphi_{1}, \varphi_{\max }\right]\right) .
\end{array}
$$

The equation for inner profile of moving scroll can be followed as

$$
\begin{array}{r}
x_{\mathrm{mi}}=-\int_{\varphi_{1}}^{\varphi}\left[\rho(\varphi)+\frac{R_{\mathrm{or}}}{2}\right] \cos \varphi \mathrm{d} \varphi \\
y_{\mathrm{mi}}=-\int_{\varphi_{1}}^{\varphi}\left[\rho(\varphi)+\frac{R_{\mathrm{or}}}{2}\right] \sin \varphi \mathrm{d} \varphi \\
\left(\varphi \in\left[\varphi_{1}, \varphi_{\max }\right]\right) .
\end{array}
$$
as

The equation for outer profile of fixed scroll can be written

$$
\begin{array}{r}
x_{\mathrm{fo}}=-\int_{\varphi_{1}}^{\varphi}\left[\rho(\varphi)-\frac{R_{\mathrm{or}}}{2}\right] \cos \varphi \mathrm{d} \varphi \\
y_{\mathrm{fo}}=-\int_{\varphi_{1}}^{\varphi}\left[\rho(\varphi)-\frac{R_{\mathrm{or}}}{2}\right] \sin \varphi \mathrm{d} \varphi \\
\left(\varphi \in\left[\varphi_{1}, \varphi_{\max }\right]\right) .
\end{array}
$$




\section{Geometrical Description of the Chamber Areas}

The intermeshed scroll wraps form a series of sealed chambers, namely, suction chamber, compression chamber, and discharge chamber, as seen in Figure 3. The volumes of these chambers are of critical importance in determining the geometrical property of a scroll compressor.

3.1. Suction Chamber. Figure 4(a) shows a pair of conjugation surfaces of a suction chamber which are positioned so they share a common center. In Figure 4(b) the moving scroll rotates around the fixed scroll at a radius of $R_{\text {or }}$ so that a suction chamber is formed at two extreme locations on the periphery. Assume the maximum tangential angle of the scroll profile is $\varphi_{\max }$ and the rotation angle of moving scroll is $\theta$. Therefore, the suction area is given by the parameters of the generation line

$$
A_{1}(\theta)=R_{\text {or }} \int_{\varphi_{\max }-\theta-2 \pi}^{\varphi_{\max }-\theta} a \varphi \mathrm{d} \varphi .
$$

3.2. Compression Chamber. The area calculation of a compression chamber is divided into two different stages.

During the first stage, the compression chamber is enclosed solely by involute of circle and the area of compression chamber can be written as follows:

$$
A_{2}(\theta)=R_{\text {or }} \int_{\varphi_{\max }-\theta-4 \pi}^{\varphi_{\max }-\theta-2 \pi} a \varphi \mathrm{d} \varphi \quad\left(0 \leq \theta \leq \frac{3 \pi}{2}-\varphi_{2}\right) .
$$

In the second stage, the compression chamber is bounded by both involutes of circle and circular arcs, as shown in Figure 5. The area of compression chamber is given by

$$
\begin{aligned}
A_{2}(\theta)= & R_{\mathrm{or}}\left(\int_{\varphi_{\max }-\theta-2 \pi}^{\varphi_{2}} R_{s} \mathrm{~d} \varphi+\int_{\varphi_{2}}^{\varphi_{\max }-\theta} a \varphi \mathrm{d} \varphi\right) \\
& +\Delta A_{2}(\theta) \quad\left(\frac{3 \pi}{2}-\varphi_{2} \leq \theta \leq \frac{3 \pi}{2}-\varphi_{1}\right),
\end{aligned}
$$

where

$$
\begin{aligned}
\Delta A_{2}(\theta)=R_{\text {or }}\left[R_{s} \sin \left(\varphi_{\max }-\theta-2 \pi-\varphi_{1}\right)-a\right] \\
\left(\frac{3 \pi}{2}-\varphi_{2} \leq \theta \leq \frac{3 \pi}{2}-\varphi_{1}\right) .
\end{aligned}
$$

When a compression chamber just opens into the discharge zone at the center, a discharge angle of $\theta^{*}$ is defined as follows:

$$
\theta^{*}=\frac{3 \pi}{2}-\varphi_{1}
$$

The volume ratio of a scroll compressor can be formulated as

$$
v=\frac{A_{1}(0)}{A_{2}\left(\theta^{*}\right)} .
$$

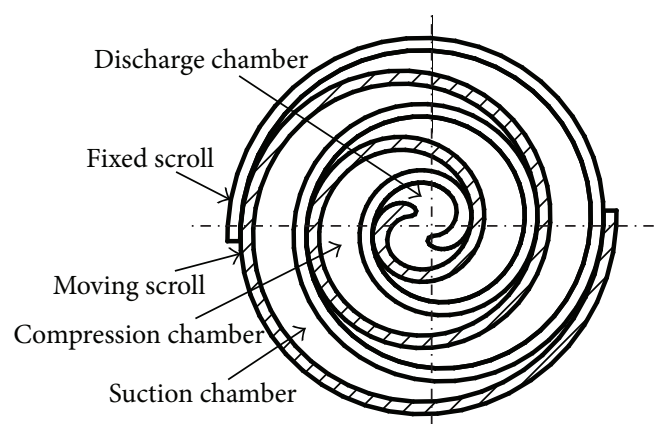

FIGURE 3: Schematic view of sealed chambers in a scroll compressor.

3.3. Discharge Chamber. The determination of a discharge area is divided into three stages in accordance with the rotation angle of the moving scroll.

In the first stage, the discharge chamber bounded by both involutes and circular curves is shown in Figure 6. The area computation of discharge chamber follows

$$
\begin{array}{r}
A_{3}(\theta)=R_{\text {or }}\left(\int_{\varphi_{2}}^{3 \pi / 2-\theta} a \varphi \mathrm{d} \varphi+\int_{\varphi_{1}}^{\varphi_{2}} R_{s} \mathrm{~d} \varphi-a\right) \\
\left(0 \leq \theta \leq \frac{3 \pi}{2}-\varphi_{2}\right) .
\end{array}
$$

During the second stage the discharge pocket is solely formed by circular curves, as can be seen in Figure 7. The area of discharge chamber is computed as follows:

$$
\begin{aligned}
A_{3}(\theta)=R_{\mathrm{or}} R_{s}\left[\left(\theta^{*}-\theta\right)-\sin \left(\theta^{*}-\theta\right)\right] \\
\qquad\left(\frac{3 \pi}{2}-\varphi_{2} \leq \theta \leq \theta^{*}\right) .
\end{aligned}
$$

At the beginning of the last stage, the largest discharge chamber formed when the rotation angle reaches discharge angle $\theta^{*}$. Consequently, the area function of discharge chamber since then could be expressed by

$$
\begin{array}{r}
A_{3}(\theta)=R_{\text {or }}\left(\int_{\varphi_{2}}^{(7 / 2) \pi-\theta} a \varphi \mathrm{d} \varphi+\int_{\varphi_{1}}^{\varphi_{2}} R_{s} \mathrm{~d} \varphi-a\right) \\
\left(\theta^{*} \leq \theta \leq 2 \pi\right) .
\end{array}
$$

\section{Dynamical Model of the Gas Forces}

The gas forces developed within scroll chambers are well known to be axial, tangential, and radial $[1,13]$. Each force varies in magnitude as the moving scroll rotates through one revolution.

4.1. Axial Force. The axial gas load is produced by the collection of gas pressures acting in the respective sealed chamber, which can be calculated by

$$
F_{a}(\theta)=\sum_{i=1}^{N} p_{i} A_{i}(\theta) \quad(0 \leq \theta \leq 2 \pi),
$$




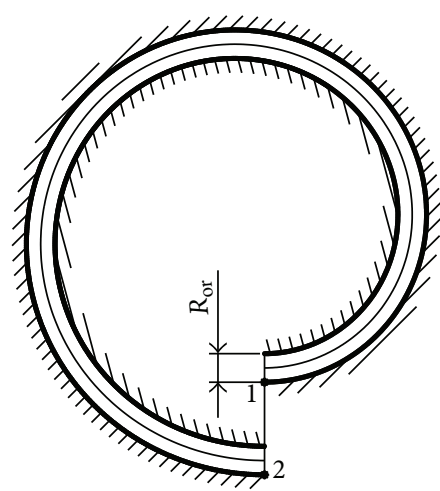

(a)

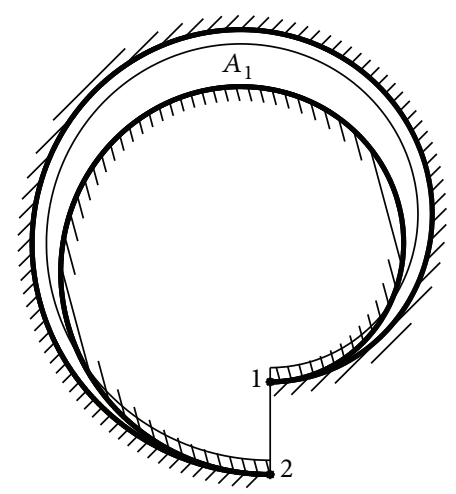

(b)

FIGURE 4: Schematic of a suction chamber.

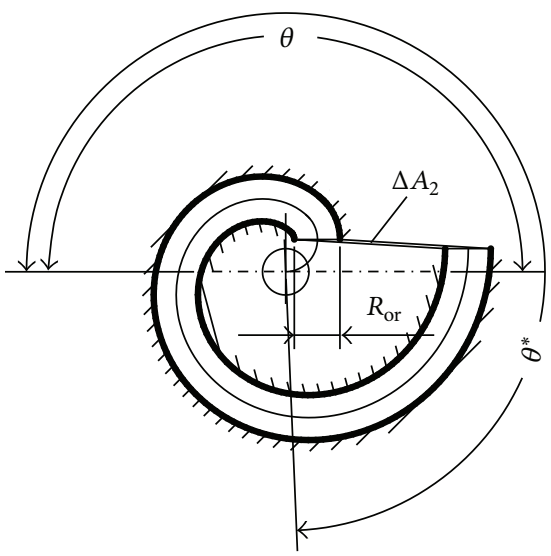

(a)

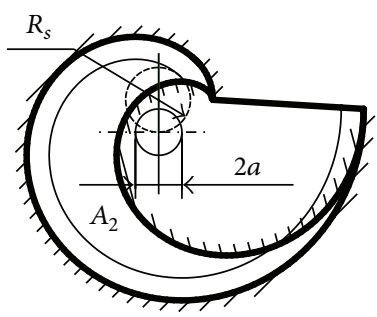

(b)

FIGURE 5: Schematic of a compression chamber.

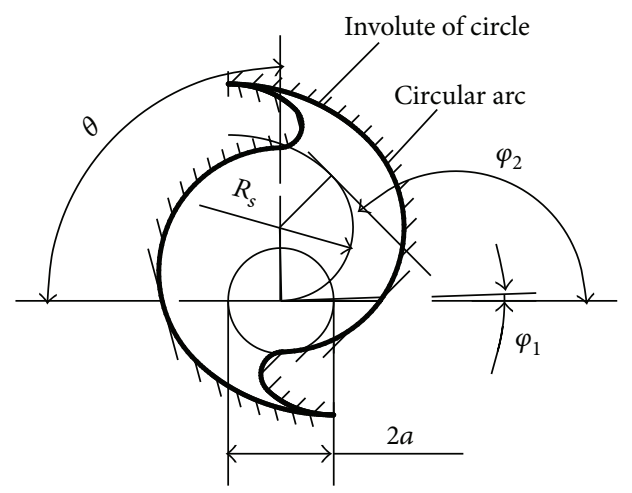

FIGURE 6: Discharge chamber bounded by two kinds of curves.

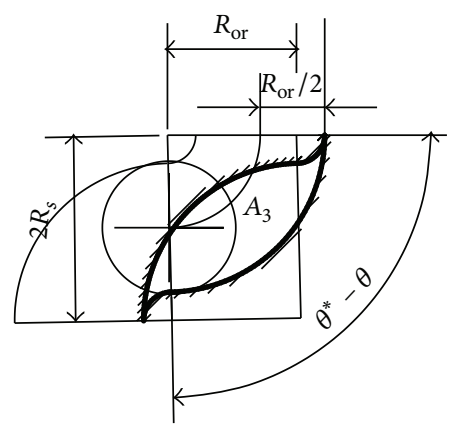

FIgURE 7: Discharge chamber bounded by circular curves.

where

$$
p_{i}=p_{s}\left[\frac{A_{1}(0)}{A_{i}(\theta)}\right]^{k} \quad(0 \leq \theta \leq 2 \pi),
$$

where $p_{s}$ indicates the suction pressure of scroll compressor and $k$ represents adiabatic index.

4.2. Tangential Force. The tangential force acts normally to the lines of flank contact points on both scrolls. There are three pairs of contacting points $1-1^{\prime}, 2-2^{\prime}$, and $3-3^{\prime}$ in the scroll compressor, and the tangential distance between each pair of the points is defined as tangential action line, which is $L_{t 1}$, $L_{t 2}$, and $L_{t 3}$, respectively, as illustrated in Figure 8. 


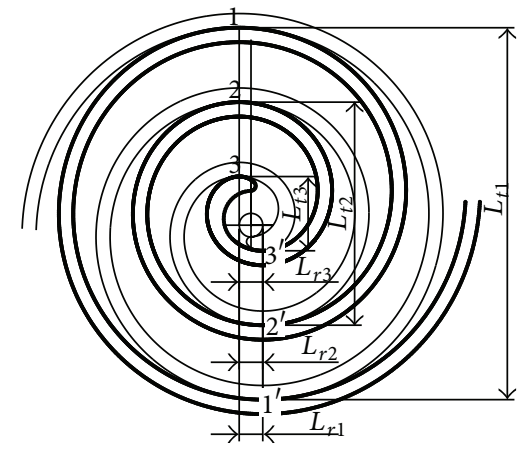

FIGURE 8: Schematic of action lines of gas force.

The tangential line $L_{t 3}$ between the innermost points 3 and $3^{\prime}$ changes in accordance with the rotation angle and can be written as

$$
\begin{aligned}
L_{t 3} & \left(0 \leq \theta \leq \frac{3 \pi}{2}-\varphi_{2}\right) \\
2 a\left(\frac{3 \pi}{2}-\theta\right) & \left(\frac{3 \pi}{2}-\varphi_{2} \leq \theta \leq \theta^{*}\right) \\
2 R_{s}\left[1-\cos \left(\theta^{*}-\theta\right)\right] & \left(\theta^{*} \leq \theta \leq 2 \pi\right) .
\end{aligned}
$$

The tangential line between other contact points can be expressed as

$$
\begin{aligned}
L_{t i}=2 a\left[\frac{3 \pi}{2} \cdot 2 \pi(N-i)-\theta\right] & \\
& (0 \leq \theta \leq 2 \pi, i=1,2) .
\end{aligned}
$$

Therefore, the tangential gas force can be obtained by

$$
F_{t}(\theta)=h \sum_{i=1}^{N} L_{t i}\left(p_{i}-p_{i+1}\right) \quad(0 \leq \theta \leq 2 \pi) .
$$

4.3. Radial Force. The radial gas force acts parallel to the lines of flank contact points on both scrolls. Radial action line $L_{r i}$ is the radial distance between the flank contact points, as indicated in Figure 8. The tangential line $L_{r 3}$ between contact points 3 and $3^{\prime}$ in discharge chamber changes with the rotation angle through one revolution. The length of radial action line $L_{r 3}$ can be given by

$$
L_{r 3}= \begin{cases}2 a & \left(0 \leq \theta \leq \frac{3 \pi}{2}-\varphi_{2}\right) \\ 2 R_{s} \sin \left(\theta^{*}-\theta\right) & \left(\frac{3 \pi}{2}-\varphi_{2} \leq \theta \leq \theta^{*}\right) \\ 2 a & \left(\theta^{*} \leq \theta \leq 2 \pi\right) .\end{cases}
$$

On the contrary, the radial action lines between contact points $2-2^{\prime}$ and $1-1^{\prime}$ are constants and can be expressed as

$$
L_{r i}=2 a \quad(0 \leq \theta \leq 2 \pi, i=1,2) .
$$

So the radial gas force can be obtained by

$$
F_{r}(\theta)=h \sum_{i=1}^{N} L_{r i}\left(p_{i}-p_{i+1}\right) \quad(0 \leq \theta \leq 2 \pi, i=1,2) .
$$

\section{Results and Discussions}

To investigate the influence of scroll profile on performance of a scroll compressor, we constructed SOC and FOC models of scroll profile for comparison. Due to the constraint of thickness of top scroll wrap, FOC models with maximum ending tangential angle $\varphi_{2} \leq 270^{\circ}$ are considered in particular. Numerical examples are performed and some results regarding geometric and dynamic characteristics of the scroll profiles will be shown herein. In carrying out the computer simulation, the following basic parameters are given based on operating condition of a $3.75 \mathrm{~kW}$ refrigeration scroll compressor: $a=2.069 \mathrm{~mm}, R_{\text {or }}=4 \mathrm{~mm}, N=3$, $h=40 \mathrm{~mm}, k=1.4, p_{s}=0.5 \mathrm{MPa}$, and $\varphi_{\max }=5.5 \pi$.

(1) Top profile has a very significant effect on the shape of scroll wrap and volume ration. As indicated in Figure 9, the central portion of the scroll wrap is thicker in FOC designs than that in SOC design. A thicker scroll wrap in general will increase the resistance to stress and deformation developed on the tip wrap. However, thicker top wrap results in a smaller volume ratio, as listed in Table 1.

(2) The axial gas force acting on moving scroll varies greatly with different top profile designs. As shown in Figure 10, the largest value of axial force occurs when rotation angle takes value of $0^{\circ}$ and at that moment the suction process is just finished. Compared to FOC designs, the axial force for SOC is increased at least by $10.54 \%$, as indicated in Table 2. Greater vibrations in axial force curves result from the FOC scroll wrap; Special attention should be paid to bearing design of scroll compressors.

(3) Tangential gas forces exerted on moving scroll with FOC and SOC profiles are compared in Figure 11 and Table 2. Like axial force, the tangential force in SOC design is larger in FOC designs. The largest value appears when discharge process begins. The maximum tangential force in SOC is at least $17.95 \%$ larger than that in FOC. Tiny vibration is found in tangential force curve in $\mathrm{FOC}_{4}$ design, while this occurrence that takes place in other FOC designs is imperceptible. The discontinuous slope coefficient of tangential action line that occurred in FOC designs is believed to cause this phenomenon. In contrast, no vibrations occur in tangential force curve in SOC design because of the continuous change of radius of curvature along the whole scroll profile.

(4) Radial forces in SOC and FOC designs are compared in Figure 12 and Table 2. The radial gas force observed in SOC scroll has smaller variation than that in the FOC ones and does not reverse. When rotation angle $3 \pi / 2 \leq \theta \leq \theta^{*}$, that is, the two scrolls mesh along scroll wraps with circular arcs, 
TABLE 1: Key geometric parameters for SOC and FOC designs.

\begin{tabular}{lcccccccc}
\hline & $\varphi_{2} /\left(^{\circ}\right)$ & $\varphi_{1} /\left(^{\circ}\right)$ & $R_{\mathrm{s}} /(\mathrm{mm})$ & $\rho\left(\varphi_{2}\right) /(\mathrm{mm})$ & $\theta^{*} /\left(^{\circ}\right)$ & $A_{2}\left(\theta^{*}\right) /\left(\mathrm{mm}^{2}\right)$ & $v$ & \multicolumn{1}{c}{$\Delta(\%)$} \\
\hline SOC & 57 & -33 & 2.069 & 2.069 & 303 & 135.4 & 5.340 & - \\
$\mathrm{FOC}_{1}$ & 90 & -25 & 2.284 & 3.249 & 295 & 141.3 & 5.202 & -2.58 \\
$\mathrm{FOC}_{2}$ & 135 & 1 & 2.877 & 4.875 & 269 & 159.9 & 4.596 \\
$\mathrm{FOC}_{3}$ & 180 & 35 & 3.579 & 6.499 & 235 & 183.8 & -13.9 \\
$\mathrm{FOC}_{4}$ & 270 & 114 & 5.094 & 9.749 & 156 & 238.5 & -30.9 \\
\hline
\end{tabular}

TABLE 2: Comparison of dynamic properties for SOC and FOC designs.

\begin{tabular}{|c|c|c|c|c|c|c|}
\hline & $F_{a \max } /(\mathrm{kN})$ & $F_{a}\left(\theta^{*}\right) /(\mathrm{kN})$ & $F_{t}\left(\theta^{*}\right) /(\mathrm{kN})$ & $F_{r \max } /(\mathrm{N})$ & $F_{r \min } /(\mathrm{N})$ & $F_{r s t} /(\mathrm{N})$ \\
\hline SOC & 2.653 & 2.333 & 5.054 & 801.1 & 705.6 & 801.1 \\
\hline $\mathrm{FOC}_{1}$ & 2.588 & 2.303 & 4.845 & 790.9 & 669.1 & 750.1 \\
\hline $\mathrm{FOC}_{2}$ & 2.406 & 2.217 & 4.285 & 742.9 & 564.9 & 617.4 \\
\hline $\mathrm{FOC}_{3}$ & 2.215 & 2.123 & 3.723 & 680.1 & 460.2 & 493.6 \\
\hline $\mathrm{FOC}_{4}$ & 1.893 & 1.954 & 2.807 & 560.7 & 301.8 & 317.3 \\
\hline
\end{tabular}

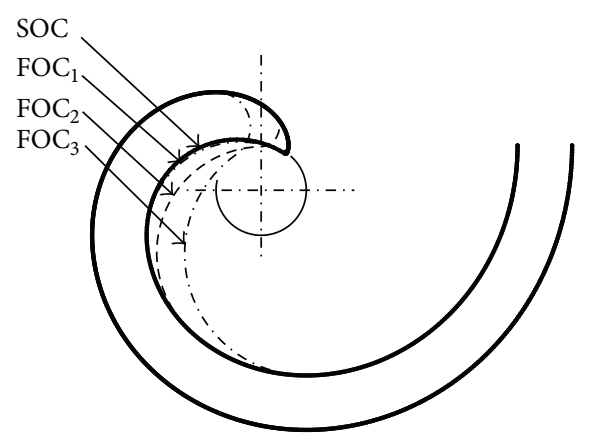

FIGURE 9: Wrap comparison of SOC and FOC profiles.

the radial force is much smaller than other rotation ranges in SOC design. Comparatively, in FOC designs the radial force developed in the sealed chamber changes periodically.

The SOC design yielded much smaller radial gas variation in the simulated computation by at least $8 \%$ as compared to the FOC ones considered. The SOC is favorable with radial gas load fluctuation rate ranging from 0 to $-13.5 \%$ while a maximum of corresponding fluctuation rate varying from 76.7 to $-4.88 \%$ is found when the $\mathrm{FOC}_{4}$ design is adopted.

This finding helps minimizing the strength and backlash requirements of the antirotation coupling, which ensures the moving scroll rotating in a plane motion, as well as reducing potential wear of mechanical components, and other effects of load reversal in the mechanism of a SOC design. Moreover, with smaller radial gas force variation a smaller radial clearance between the moving and fixed scrolls can be achieved, which leads to an optimal design of scroll compressor in terms of leakage loss, bearing life, and reliability.

\section{Conclusion}

This paper proposed a way to redesign the geometry of scroll wrap in which the central and main part of the profile

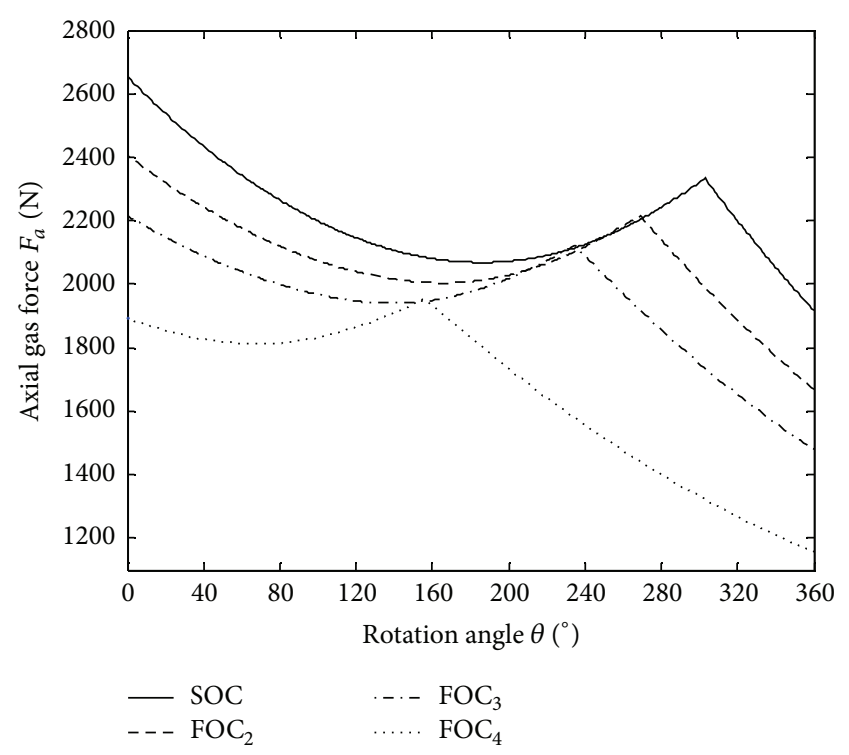

FIgURE 10: Axial gas force.

can be expressed in a general form. This work is essential for the profile design of scroll components to ensure good performance of scroll compressors. Area estimations are given for the sealed chambers formed during working process of a scroll compressor. Afterwards, the gas force acting on the moving scroll is calculated. The geometric and dynamic models have been validated by numerical simulation of a scroll compressor. For comparison purposes some simulation examples for SOC and FOC profile designs are presented. As a result of the above derivation and simulation, the following conclusions can be drawn with regard to the proposed scroll profile designs.

(1) This study intends to identify the geometrical and dynamical characteristics of SOC and FOC scroll profiles 


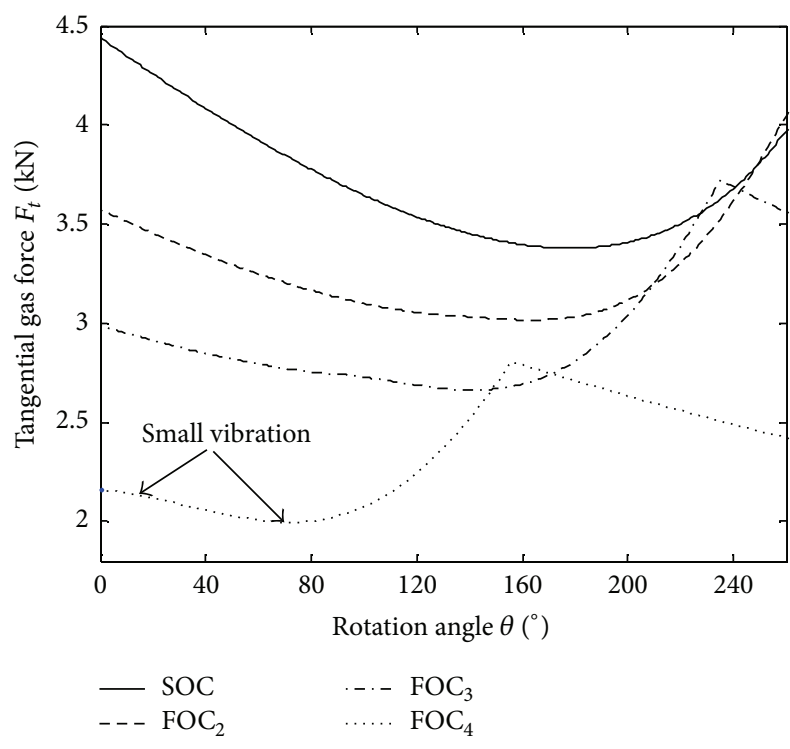

FIGURE 11: Tangential gas force.

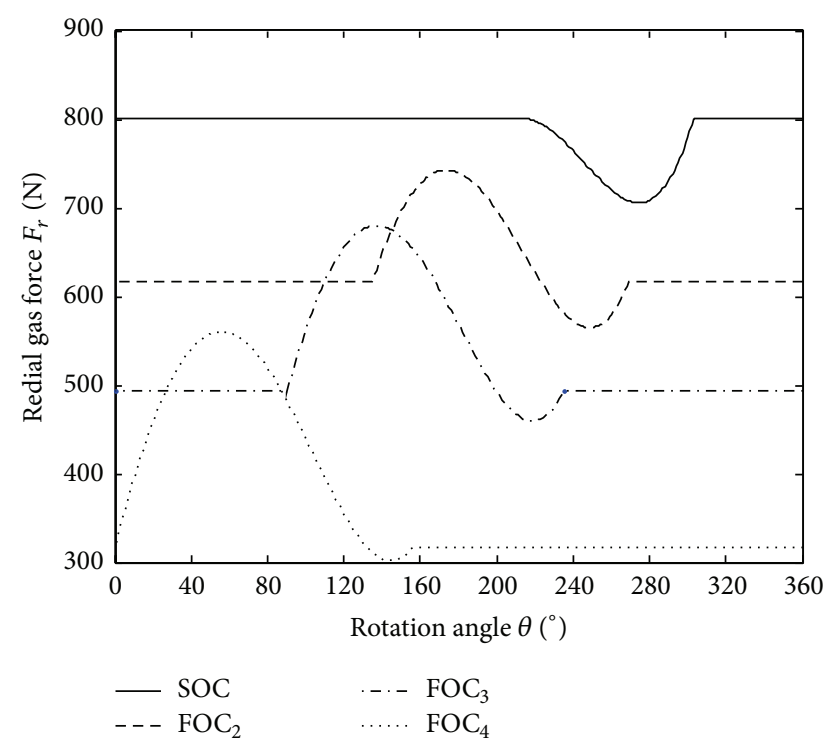

Figure 12: Radial gas force.

\section{Nomenclature}

$\varphi$ : $\quad$ Tangential angle

$\varphi_{1}$ : $\quad$ Starting tangential angle of circular arc

$\varphi_{2}$ : Ending tangential angle of circular arc

$\varphi_{\max }:$ Maximum tangential angle of scroll profile

$R_{s}: \quad$ Radius of circular arc

$a$ : Radius of the base circle of involute

$R_{\text {or: }}$ Rotation radius

$\rho: \quad$ Radius of curvature of scroll profile

$\theta: \quad$ Rotation angle of moving scroll

$A_{1}$ : Area of suction chamber

$A_{2}$ : Area of compression chamber

$A_{3}$ : Area of discharge chamber

$\theta^{*}: \quad$ Discharge angle

$v$ : Volume ratio

$\Delta v: \quad$ Increment volume ratio

$F_{a}$ : Axial gas load

$p_{s}$ : Suction pressure

$\kappa: \quad$ Adiabatic index

$N$ : Number of pairs of chambers

$F_{t}$ : Tangential gas load

$L_{t i}$ : Length of tangential action line

$h$ : Height of scroll wrap

$F_{r}$ : Radial gas load

$L_{r i}$ : Length of radial action line

$F_{r \max }$ : Maximum radial gas load

$F_{\text {rmin }}$ : Minimum radial gas load

$F_{r s t}: \quad$ Stable radial gas load.

Subscripts

a: Axial

$t$ : Tangential

$r$ : Radial

$s: \quad$ Suction

st: Stable

Or: Orbiting

max: Maximum

min: Minimum

$i$ : $\quad$ Inner

$o: \quad$ Outer

$m$ : Moving

$f$ : $\quad$ Fixed.

\section{Conflict of Interests}

The authors declare that there is no conflict of interests regarding the publication of this paper.

\section{Acknowledgments}

The authors thank Professor Liu Zhenquan for valuable discussions. This study is supported by National Natural Science Foundation of China (Grant no. 51265027) and The Fundamental Research Funds for the Universities in Gansu Province (Grant no. 1302ZTC034). 


\section{References}

[1] E. Morishita and M. Sugihara, "Some design problems of scroll compressor," Bulletin of the JSME, vol. 29, no. 258, pp. 41394146, 1986.

[2] Q. Jianguo and T. Wen, "Numerical modelling of temperature distribution for orbiting scroll wrap in an air scroll compressor," International Journal of Heat and Mass Transfer, vol. 67, pp. 678689, 2013.

[3] M. M. Cui, "Numerical study of unsteady flows in a scroll compressor," ASME Journal of Fluids Engineering, vol. 128, no. 5, pp. 947-955, 2006.

[4] Z. Jiang, D. K. Harrison, and K. Cheng, "Computer-aided design and manufacturing of scroll compressors," Journal of Materials Processing Technology, vol. 138, no. 1-3, pp. 145-151, 2003.

[5] E. Winandy, C. S. Saavedra O, and J. Lebrun, "Experimental analysis and simplified modelling of a hermetic scroll refrigeration compressor," Applied Thermal Engineering, vol. 22, no. 2, pp. 107-120, 2002.

[6] D. A. Cha, O. K. Kwon, and M. D. Oh, "An experimental study on semiconductor process chiller using the digital scroll compressor," Journal of Mechanical Science and Technology, vol. 28, no. 8, pp. 3345-3352, 2014.

[7] Y. Liu, C. Hung, and Y. Chang, "Study on involute of circle with variable radii in a scroll compressor," Mechanism and Machine Theory, vol. 45, no. 11, pp. 1520-1536, 2010.

[8] Z. Liu, G. Du, Z. Qi, and J. Gu, "The conjugacy analysis of modified part of scroll profiles," in Proceedings of the International Compressor Engineering Conference at Purdue, pp. 479484, West Lafayette, Ind, USA, 1994.

[9] Y.-R. Lee and W.-F. Wu, "On the profile design of a scroll compressor," International Journal of Refrigeration, vol. 18, no. 5, pp. 308-317, 1995.

[10] J. W. Bush and W. P. Beagle, "Derivation of a general relation governing the conjugacy of scroll profiles," in Proceedings of the International Compressor Engineering Conference at Purdue, pp. 1079-1087, 1992.

[11] J. Gravesen and C. Henriksen, "The geometry of the scroll compressor," SIAM Review, vol. 43, no. 1, pp. 113-126, 2001.

[12] B. Blunier, G. Cirrincione, Y. Hervé, and A. Miraoui, "A new analytical and dynamical model of a scroll compressor with experimental validation," International Journal of Refrigeration, vol. 32, no. 5, pp. 874-891, 2009.

[13] Y. Chen, N. P. Halm, E. A. Groll, and J. E. Braun, "Mathematical modeling of scroll compressors-part I: compression process modeling," International Journal of Refrigeration, vol. 25, no. 6 , pp. 731-750, 2002. 


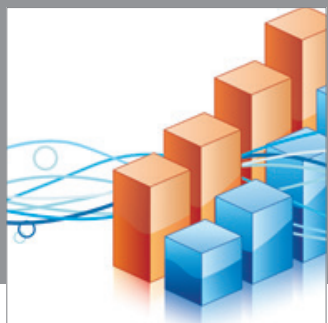

Advances in

Operations Research

mansans

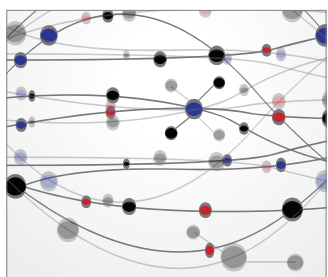

The Scientific World Journal
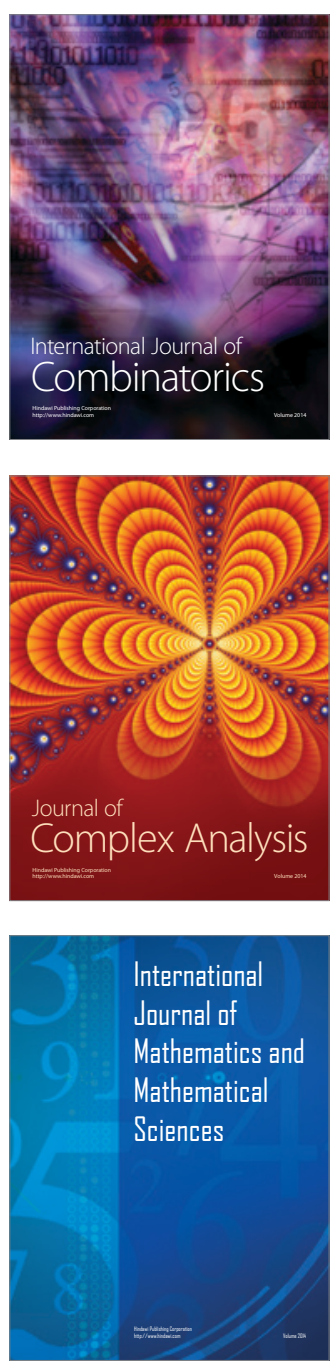
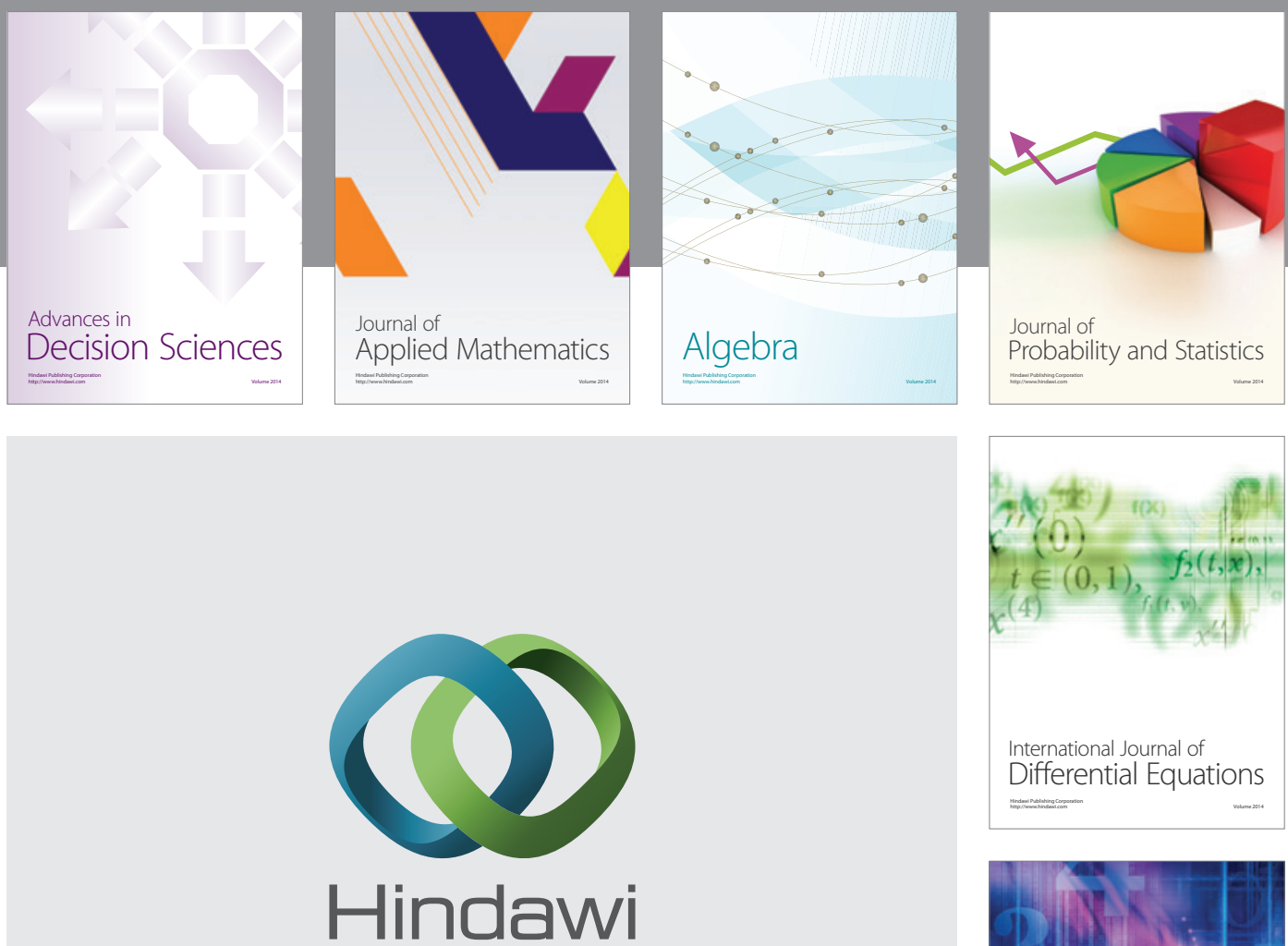

Submit your manuscripts at http://www.hindawi.com
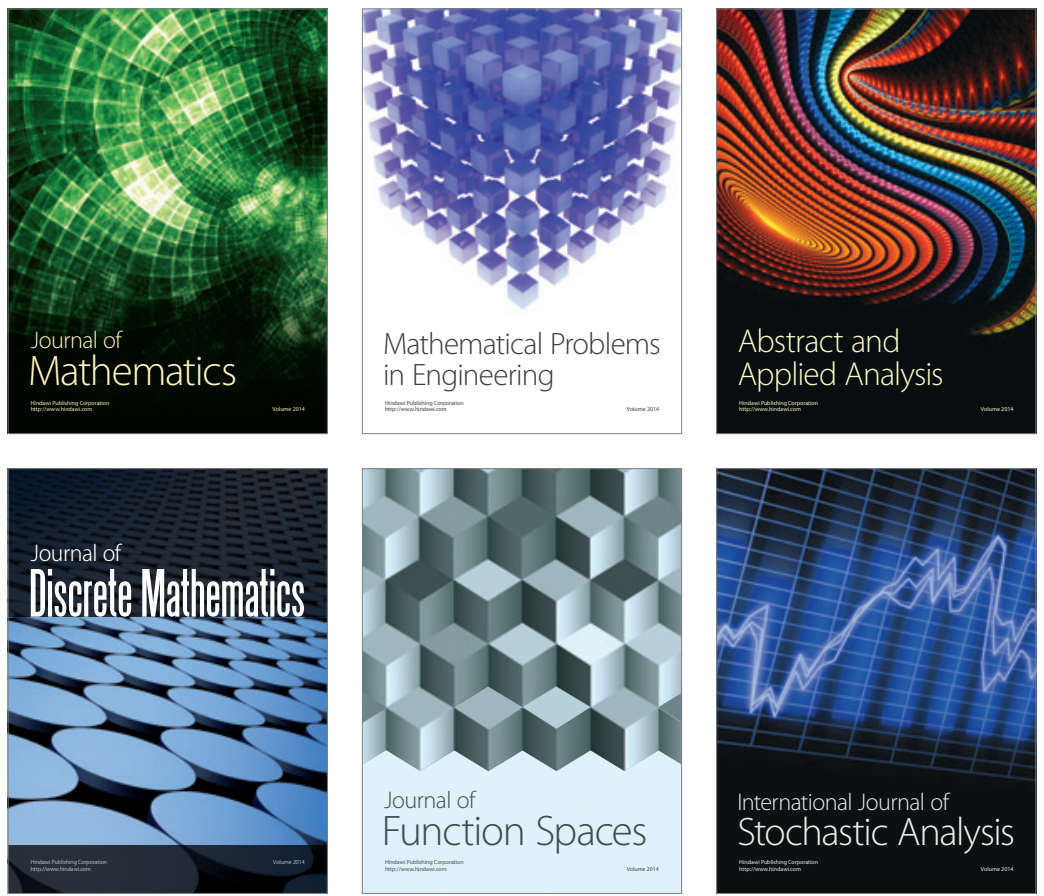

Journal of

Function Spaces

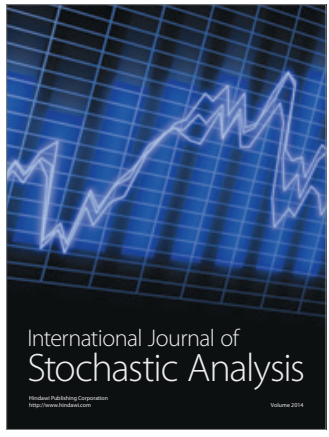

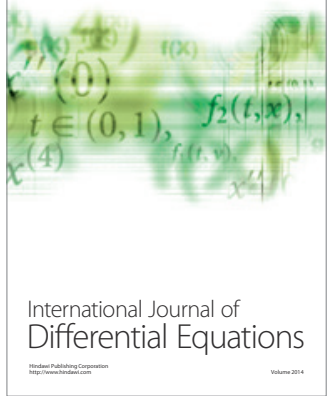
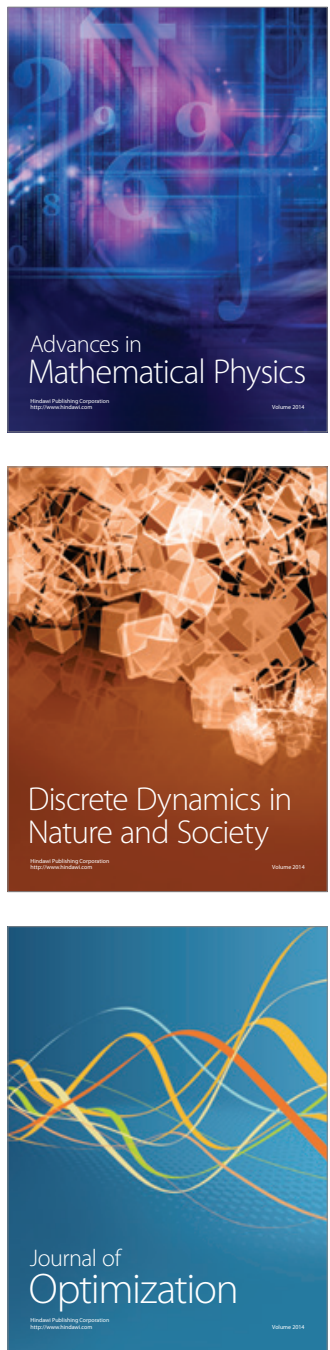